\title{
3 Research Square \\ Comparison of Different Types of Therapy for Overactive Bladder: A Systematic Review and Network Meta-Analysis
}

\section{Peng Liu}

Qilu Hospital, Shandong University

\section{Lei Sheng}

Qilu Hospital, Shandong University

\section{Yan Li}

Qilu Hospital, Shandong University

\section{Benkang Shi}

Qilu Hospital, Shandong University

\section{Hongda Guo}

Qilu Hospital, Shandong University

\section{Qiujie Zhang}

Shandong University

Hu Guo ( $\sim$ guohu97@sdu.edu.cn )

Qilu Hospital, Shandong University

\section{Research Article}

Keywords: overactive bladder, randomized controlled trials, Mirabegron and PTNS, OAB syndrome

Posted Date: December 10th, 2021

DOI: https://doi.org/10.21203/rs.3.rs-1127659/v1

License: (c) (i) This work is licensed under a Creative Commons Attribution 4.0 International License. Read Full License 


\section{Abstract}

To compare the efficacy and safety of different interventions (including Antimuscarinics, Mirabegron, OnabotulinumtoxinA, sacral neuromodulation (SNM) and peripheral tibial nerve stimulation (PTNS)) on idiopathic overactive bladder (OAB). PubMed, Embase, Cochrane library, and other sources were searched for randomized controlled trials (RCTs) comparing interventions of OAB from 1 January 2000 to 19 April 2021. A systematic review and network meta-analysis were performed by two authors independently. 55 RCTs involving 32,507 patients were included in this network. Overall, Antimuscarinics, Mirabegron, OnabotulinumtoxinA, SNM, and PTNS were more efficacious than placebo and SNM showed the best effect for reducing micturition frequency, urgency episodes and urgency urinary incontinence episodes. OnabotulinumtoxinA was the best in achieving reduction of $100 \%$ and $\geq 50 \%$ in the number of urinary incontinence episodes / day and PTNS was the best in reducing urinary incontinence episodes. The efficacy of Antimuscarinics, Mirabegron and PTNS were similar in reducing micturition frequency, urinary incontinence episodes and urgency urinary incontinence episodes. The results revealed that all interventions included in the network were efficacious in managing adult OAB syndrome compared with placebo. Furthermore, SNM and OnabotulinumtoxinA were the most efficient treatments for overactive bladder.

\section{Introduction}

Overactive bladder (OAB) was defined in 2002 by the International Continence Society as a storage symptom concerning "urgency, with or without urgency urinary incontinence, in the absence other obvious pathology"[1]. The Epidemiology of Incontinence (EPIC) study suggested that the incidence rate of OAB is high in both men and women ( $10.8 \%$ and $12.8 \%$, respectively) $[2,3]$. OAB impairs the health-related quality of life (HRQoL) and creates a significant economic burden. The cost of urinary incontinence is reported to account for about 2 percent of total health care costs in the United States each year[4]. The American Urological Association (AUA) guidelines recommend behavioral therapy as the first line treatment. If behavioral therapy doesn't work, oral Antimuscarinics and Mirabegron are recommended as second-line treatment. When patients are refractory to first- and second-line OAB treatments, clinicians can provide third-line therapy, including OnabotulinumtoxinA, SNM and PTNS $[3,5,6]$. This therapeutic modality was derived by balancing the potential benefits to the patient with the risks including invasiveness of the treatment, the duration and severity of potential adverse events and the reversibility of potential adverse events. Although risks are difficult to be accessed, the benefits are easy to assess because of uniform outcome indicators. There are many RCTs comparing the efficacy of those interventions for $\mathrm{OAB}$, but most of them focus on drug therapy, such as Antimuscarinics, Mirabegron and very few involving SNM, PTNS, which leads to inadequate awareness among clinicians about the relative effectiveness of these treatments. Direct comparisons as well as network meta-analysis that included several of these interventions have been performed[7, 8], but no network meta-analysis was conducted to evaluated all these treatments. Therefore, in this study, we intend to perform a systematic review and 
network meta-analysis to guide clinical practice by comparing the relative effectiveness of different interventions for the treatment of adults with $O A B$

\section{Methods}

This network meta-analysis was conducted on the basis of the PRISMA extension statement for network meta-analysis [9]. This study was registered with PROSPERO (CRD42021251966).

\section{Data sources and searches}

PubMed, Embase, Cochrane library, and other sources were searched to find relevant articles published from 1 January 2000 to 19 April 2021. For an outcome in the same trial, only the data closest to the 12week follow-up were extracted. The detailed search strategy is shown in Supplemental Table S1.

\section{Eligibility criteria}

We included RCTs reported in English comparing Anticholinergics, Mirabegron, OnabotulinumtoxinA, SNM, PTNS with each other or placebo, in adults with OAB. The inclusion criteria and exclusion criteria were summarized in Supplemental Table S2.

\section{Data extraction}

Two investigators $(\mathrm{PL}, \mathrm{YL})$ independently extracted data using a unified data extraction form, and difference was resolved by another reviewer (LS). We recorded the outcomes as close to 12 weeks as possible. The primary outcome was mean change in the frequency of micturition/day on the basis of European Medicines Agency and Food and Drug Administration guidelines for OAB trials. Secondary outcomes were proportions of patients achieving $100 \%$ and $\geq 50 \%$ reductions from baseline in urinary incontinence episodes /day, mean change in urgency episodes/day, mean change in urgency urinary incontinence episodes /day, mean change in urinary incontinence episodes /day. Main characteristics of qualified trials such as first author, publication year, methods, number of patients, inclusion and exclusion criteria, interventions and outcomes were extracted to conduct further analysis.

\section{Risk of bias assessment}

The bias risk of included RCTs was assessed using the RoB (Cochrane risk-of-bias tool for randomized trials) tool consisting of randomization process, deviations from intended interventions, missing outcome data, measurement of the outcome, and selection of the reported result. Two independent researchers (PL and $\mathrm{YL}$ ) assessed risk of bias of all included studies according to RoB. Any inconsistency was discussed and resolved by LS to reach an agreement. A risk of bias graph is shown in Supplemental Figure S1. 


\section{Data synthesis and analysis}

Forest plots of pairwise meta-analyses were accomplished by Stata 16.0 [10]. We assessed network connectivity using a network diagram (Stata, version 16.0). Network meta-analysis was conducted in a Bayesian framework within the GEMTC package in the R-Statistics [11] and the J.A.G.S. program as previously described[12]. Consistent and inconsistent models were considered and compared using deviance information criteria [13] (Supplemental Table S3). If the difference between them is less than 5, consistent fix-effect model was selected. Otherwise, random-effect model was selected. We used an unrelated mean effects model to assess inconsistency by comparing the model fit and between-study variance (heterogeneity) estimate of the pair-wise comparisons against the results of the consistency model. Network consistency was assessed by comparing direct evidence and indirect evidence for each comparison using a node-splitting technique $[14,15]$. The assessment of heterogeneity was performed by the $\mathrm{R}$ package 'meta', which is represented by $\mathrm{I}^{2}$ ranging between $0 \%$ and $100 \%$. The ranking probabilities were also calculated using $\mathrm{R}$, and the broken line graph was generated by GraphPad Prism.

\section{Sensitivity analysis}

Sensitivity analysis was performed to test the robustness and reliability of results by excluding studies deemed to have a high risk of bias.

\section{Results}

\section{Included studies}

The literature search identified 1,707 unique references, and 1,409 studies were excluded during screening (Figure 1). Of the 298 full-text articles assessed for eligibility, 243 were excluded. Overall, 55 studies ( $n=$ $32,507)$ were included in the network meta-analysis. Summaries of all included studies are shown in Supplemental Table S4.

\section{Pairwise meta-analyses result for different endpoints}

Pairwise meta-analyses results were shown in Supplemental Figure S2. The results suggested that all treatments were more efficacious than placebo in reducing micturition frequency, urinary incontinence episodes, urgency episodes, and urgency urinary incontinence episodes. Patients receiving Antimuscarinics and Mirabegron showed no significant difference in reducing micturition frequency, urinary incontinence episodes, urgency episodes, and urgency urinary incontinence episodes. There was no difference in reducing micturition frequency and urgency urinary incontinence episodes between patients treated with Antimuscarinics and PTNS, and there was no difference in reducing urgency urinary incontinence episodes between patients treated with SNM and OnabotulinumtoxinA. In terms of $100 \%$ and $\geq 50 \%$ reductions from baseline in urinary incontinence episodes /day, there was no significant 
difference in the efficacy of placebo, Antimuscarinics and Mirabegron. Other direct comparisons were of limited significance due to the insufficient number of studies.

\section{Network Meta-Analysis on the Outcomes of Interests}

The primary network including all studies (regardless of RoB) for all outcomes were presented in Figure 2.

The network meta-analysis (Figure 3) indicated that treatment with OnabotulinumtoxinA or SNM resulted in obviously greater mean reductions in micturition frequency, urinary incontinence episodes, urgency episodes, and urgency urinary incontinence episodes compared with all of the other interventions included in the network and the efficacy of Antimuscarinics, Mirabegron and PTNS were similar and were better than that of placebo. In addition, the results suggested that patients receiving OnabotulinumtoxinA have the highest odds of achieving reduction of $100 \%$ and $\geq 50 \%$ in the number of urinary incontinence episodes / day [odds ratios relative to placebo: 5.92 (95\% Crl 3.53-10.29) and 3.57 (95\% Crl 2.56-5.00)]. Also, Antimuscarinics or Mirabegron was superior to placebo in $100 \%$ and $\geq 50 \%$ reductions from baseline in urinary incontinence episodes / day.

\section{Rank probabilities}

The Bayesian ranking probabilities of comparable treatments in different populations are shown in Figure 4. In overall, SNM was most likely to be ranked first for reducing micturition frequency, urgency episodes and urgency urinary incontinence episodes, and placebo ranked lowest. The ranking results for urinary frequency reduction and urgency urinary incontinence episodes reduction was as follows: SNM ranked first, OnabotulinumtoxinA ranked second, PTNS ranked third, one of Mirabegron and Antimuscarinics ranked fourth and the other ranked fifth, and placebo ranked sixth. The ranking results for urgency episodes reduction was as follows: SNM ranked first, OnabotulinumtoxinA ranked second, Antimuscarinics ranked third, Mirabegron ranked fourth, and placebo ranked fifth. The ranking results for urinary incontinence episodes reduction was as follows: SNM, OnabotulinumtoxinA and PTNS were the top three in no particular order, Antimuscarinics ranked fourth, Mirabegron ranked fifth, and placebo ranked sixth. The ranking results for achieving reduction of $100 \%$ and $\geq 50 \%$ in the number of urinary incontinence episodes / day were as follows: OnabotulinumtoxinA ranked first, SNM ranked second, and placebo ranked lowest.

\section{Consistency and inconsistency assessment}

The fit of the consistency model in all comparisons was similar or better than the fit of the inconsistency model (Supplemental Table S3). Node splitting analysis was performed to evaluate consistencies by comparing differences between the direct and indirect evidence and the result was shown in Supplemental Table S5, which showed no significant differences in most comparisons except for placebo 
vs OnabotulinumtoxinA, Anticholinergics vs SNM, OnabotulinumtoxinA vs SNM in the comparison of micturition, urinary incontinence episodes and $100 \%$ reductions from baseline in urinary incontinence episodes / day.

\section{Sensitivity analyses}

The results of sensitivity analysis (Supplemental Figure S3 and S4) excluding 12 studies considered to have a high RoB showed little impact on the results of the network meta-analysis. The main change of sensitivity analysis was summarized in Supplemental Table S6.

\section{Discussion}

In the absence of a direct head-to-head comparison of all second line and third line treatments recommend by AUA for adult OAB symptoms, the present detailed systemic review and network metaanalysis is the first review to compare the relative effectiveness of all those interventions. Six efficacy outcomes were assessed, with each network including between 7 and 52 studies.

Results from pairwise meta-analysis revealed that all five interventions were more efficacious than placebo with regard to the outcomes included in this study. Results from network meta-analysis indicated that OnabotulinumtoxinA and SNM were more efficacious than all of the other interventions in reducing micturition frequency, urinary incontinence episodes, urgency episodes and urgency urinary incontinence episodes. The efficacy of Antimuscarinics, Mirabegron and PTNS was similar. In terms of reduction of $100 \%$ and $\geq 50 \%$ in the number of urinary incontinence episodes /day, OnabotulinumtoxinA had the best effect.

There are insufficient studies on Treatment Emergent Adverse Events (TEAEs), and the TEAEs of different interventions are inconsistent[16], hence the present network meta-analysis was not performed. A systematic review on TEAEs was conducted. The most common TEAEs of Antimuscarinics were dry mouth and constipation[17], which seriously affected patient compliance. Mirabegron caused an increase in blood pressure that was positively correlated with the duration of the drug[18, 19]. Therefore, Mirabegron is not recommended in patients with severe uncontrolled hypertension or end-stage renal disease. OnabotulinumtoxinA can cause urinary tract infections and retention of urine[8,20], sometimes requiring catheterization to empty the bladder. TEAEs of SNM were pain at the stimulator and lead sites, lead migration, infection, and the requirement for surgical revision[21]. Although some patients had discomfort and mild pain at the site of stimulation, as well as tingling and acid swelling of the leg, PTNS was safe.

In terms of cost, all third-line treatments are more expensive than drug treatments, and SNM is considered to be the most expensive treatment compared with other interventions in the short term[22-24], which may be an important factor affecting its application. However, SNM seems to be either cost saving, or 
acceptably cost effective compared with ongoing medical therapy, PTNS, or OnabotulinumtoxinA in the long term[25].

There were several limitations to the current network meta-analysis study. The current study primarily compared short-term efficacy at 12 weeks follow-up and we also extracted outcome data closest to the 12-week follow-up in this study, resulting in the lack of comparison of long-term efficacy. Further research is needed to evaluate the long-term efficacy of these interventions. In addition, placebo differed in their mode of administration. In the OnabotulinumtoxinA trials, placebo was administered as a single injection, and in the mirabegron and anticholinergics trials, the placebos were administered as daily oral tablets, while in the SNM and PTNS experiment, sham stimulation was used as placebo. To link these interventions of interest into a network, we hypothesized that different placebo treatments were equally effective in the same population. However, one study [26] found that different administrations affect the relative efficacy of placebo for osteoarthritis of the knee, this is a limitation of this network meta-analysis.

This network meta-analysis further demonstrates the relative effectiveness of all five interventions included in this network in treating OAB. Among them, SNM was found to have the best efficacy and fewer side effects. Although behavioral therapy and drug therapy were the first- and second- line treatment for $O A B$, the overall treatment effect was usually small. Because of limited efficacy and severe adverse effect, some patients were refractory to behavioral therapy and medicine treatment. Therefore, it is reasonable to consider third line treatment as an option due to better relative efficacy in patients who have not benefit from medicine treatment. SNM is especially worth developing because of its better relative efficacy and fewer adverse effect.

\section{Conclusions}

This network meta-analysis revealed that SNM and OnabotulinumtoxinA achieved the best results in most outcomes at 12 weeks follow-up. As there is a lack of head-to-head comparison studies among Anticholinergics, Mirabegron, OnabotulinumtoxinA, SNM, PTNS for the treatment of adult OAB symptoms, the present network meta-analysis provides the best available evidence for comparing of these five interventions. Meanwhile, well-designed and head-to-head RCTs are needed to assess efficacy and TEAEs of interventions in managing $\mathrm{OAB}$.

\section{Declarations}

\section{Acknowledgements}

None.

\section{Author contributions}

All the authors have made substantial contributions to the conception of the study. P. L., Y. L., B. S., H. G. and H. G. contributed to designing the study. P. L., L. S. and Q. Z. contributed to acquisition and analysis 
of data. P. L. and L. S. contributed to interpretation of data. P. L., L. S. and Y. L. contributed to drafting of the manuscript. P. L. and L. S. contributed to critical revision of the manuscript. All authors approved the final manuscript.

\section{Funding}

We did not receive any funding.

\section{Competing interests}

The authors declare no competing interests.

\section{Footnotes}

These authors contributed equally: Peng Liu and Lei Sheng.

\section{References}

1. Peyronnet, B. et al. A Comprehensive Review of Overactive Bladder Pathophysiology: On the Way to Tailored Treatment. European urology 75, 988-1000, doi:10.1016/j.eururo.2019.02.038 (2019).

2. Irwin, D. E. et al. Population-based survey of urinary incontinence, overactive bladder, and other lower urinary tract symptoms in five countries: results of the EPIC study. European urology 50, 1306-1314; discussion 1314-1305, doi:10.1016/j.eururo.2006.09.019 (2006).

3. Lightner, D. J., Gomelsky, A., Souter, L. \& Vasavada, S. P. Diagnosis and Treatment of Overactive Bladder (Non-Neurogenic) in Adults: AUA/SUFU Guideline Amendment 2019. J Uro/ 202, 558-563, doi:10.1097/ju.0000000000000309 (2019).

4. Reeves, P. et al. The current and future burden and cost of overactive bladder in five European countries. European urology 50, 1050-1057, doi:10.1016/j.eururo.2006.04.018 (2006).

5. De Nunzio, C. et al. Beyond Antimuscarinics: A Review of Pharmacological and Interventional Options for Overactive Bladder Management in Men. European urology 79, 492-504, doi:10.1016/j.eururo.2020.12.032 (2021).

6. Raju, R. \& Linder, B. J. Evaluation and Treatment of Overactive Bladder in Women. Mayo Clinic proceedings 95, 370-377, doi:10.1016/j.mayocp.2019.11.024 (2020).

7. Drake, M. J. et al. Comparative assessment of the efficacy of onabotulinumtoxinA and oral therapies (anticholinergics and mirabegron) for overactive bladder: a systematic review and network metaanalysis. BJU international 120, 611-622, doi:10.1111/bju.13945 (2017).

8. Lo, C. W., Wu, M. Y., Yang, S. S., Jaw, F. S. \& Chang, S. J. Comparing the Efficacy of OnabotulinumtoxinA, Sacral Neuromodulation, and Peripheral Tibial Nerve Stimulation as Third Line Treatment for the Management of Overactive Bladder Symptoms in Adults: Systematic Review and Network Meta-Analysis. Toxins 12, doi:10.3390/toxins12020128 (2020). 
9. Hutton, B. et al. The PRISMA extension statement for reporting of systematic reviews incorporating network meta-analyses of health care interventions: checklist and explanations. Annals of internal medicine 162, 777-784, doi:10.7326/m14-2385 (2015).

10. Bagos, P. G. Meta-analysis in Stata using gllamm. Research synthesis methods 6, 310-332, doi:10.1002/jrsm.1157 (2015).

11. Hu, D., O'Connor, A. M., Wang, C., Sargeant, J. M. \& Winder, C. B. How to Conduct a Bayesian Network Meta-Analysis. Frontiers in veterinary science 7, 271, doi:10.3389/fvets.2020.00271 (2020).

12. Sheng, L. et al. Selection of optimal first-line immuno-related therapy based on specific pathological characteristics for patients with advanced driver-gene wild-type non-small cell lung cancer: a systematic review and network meta-analysis. Therapeutic advances in medical oncology 13, 17588359211018537, doi:10.1177/17588359211018537 (2021).

13. Dias, S., Sutton, A. J., Welton, N. J. \& Ades, A. E. Evidence synthesis for decision making 3 : heterogeneity-subgroups, meta-regression, bias, and bias-adjustment. Medical decision making : an international journal of the Society for Medical Decision Making 33, 618-640, doi:10.1177/0272989x13485157 (2013).

14. Dias, S. et al. in NICE DSU Technical Support Document 4: Inconsistency in Networks of Evidence Based on Randomised Controlled Trials (National Institute for Health and Care Excellence (NICE) Copyright (C) 2014 National Institute for Health and Clinical Excellence, unless otherwise stated. All rights reserved., 2014).

15. Dias, S., Welton, N. J., Caldwell, D. M. \& Ades, A. E. Checking consistency in mixed treatment comparison meta-analysis. Statistics in medicine 29, 932-944, doi:10.1002/sim.3767 (2010).

16. Hsiao, S. M. \& Lin, H. H. Medical treatment of female overactive bladder syndrome and treatmentrelated effects. Journal of the Formosan Medical Association = Taiwan yi zhi 117, 871-878, doi:10.1016/j.jfma.2018.01.011 (2018).

17. Andersson, K. E. Antimuscarinics for treatment of overactive bladder. The Lancet. Neurology 3, 46-53, doi:10.1016/s1474-4422(03)00622-7 (2004).

18. Wagg, A. et al. The efficacy and tolerability of the $\beta 3$-adrenoceptor agonist mirabegron for the treatment of symptoms of overactive bladder in older patients. Age and ageing 43, 666-675, doi:10.1093/ageing/afu017 (2014).

19. Chapple, C. R. et al. Safety and Efficacy of Mirabegron: Analysis of a Large Integrated Clinical Trial Database of Patients with Overactive Bladder Receiving Mirabegron, Antimuscarinics, or Placebo. European urology 77, 119-128, doi:10.1016/j.eururo.2019.09.024 (2020).

20. Clyne, M. Incontinence: OnabotulinumtoxinA safer than abobotulinumtoxinA for OAB. Nature reviews. Urology 10, 253, doi:10.1038/nrurol.2013.64 (2013).

21. De Wachter, S., Vaganee, D. \& Kessler, T. M. Sacral Neuromodulation: Mechanism of Action. European urology focus 6, 823-825, doi:10.1016/j.euf.2019.11.018 (2020).

22. Arlandis, S. et al. Cost-effectiveness of sacral neuromodulation compared to botulinum neurotoxin a or continued medical management in refractory overactive bladder. Value in health : the journal of 
the International Society for Pharmacoeconomics and Outcomes Research 14, 219-228, doi:10.1016/j.jval.2010.08.006 (2011).

23. Barnett, G. \& Ockrim, J. Re: Cost of neuromodulation therapies for overactive bladder: percutaneous tibial nerve stimulation versus sacral nerve stimulation: M. Martinson, S. MacDiarmid and E. Black J Urol 2013; 189: 210-216. J Urol 190, 1444-1445, doi:10.1016/j.juro.2013.04.131 (2013).

24. Siddiqui, N. Y., Amundsen, C. L., Visco, A. G., Myers, E. R. \& Wu, J. M. Cost-effectiveness of sacral neuromodulation versus intravesical botulinum $A$ toxin for treatment of refractory urge incontinence. J Uro/ 182, 2799-2804, doi:10.1016/j.juro.2009.08.031 (2009).

25. Marcelissen, T. et al. Management of Idiopathic Overactive Bladder Syndrome: What Is the Optimal Strategy After Failure of Conservative Treatment? European urology focus 4, 760-767, doi:10.1016/j.euf.2018.05.004 (2018).

26. Bannuru, R. R. et al. Effectiveness and Implications of Alternative Placebo Treatments: A Systematic Review and Network Meta-analysis of Osteoarthritis Trials. Annals of internal medicine 163, 365-372, doi:10.7326/m15-0623 (2015).

\section{Figures}




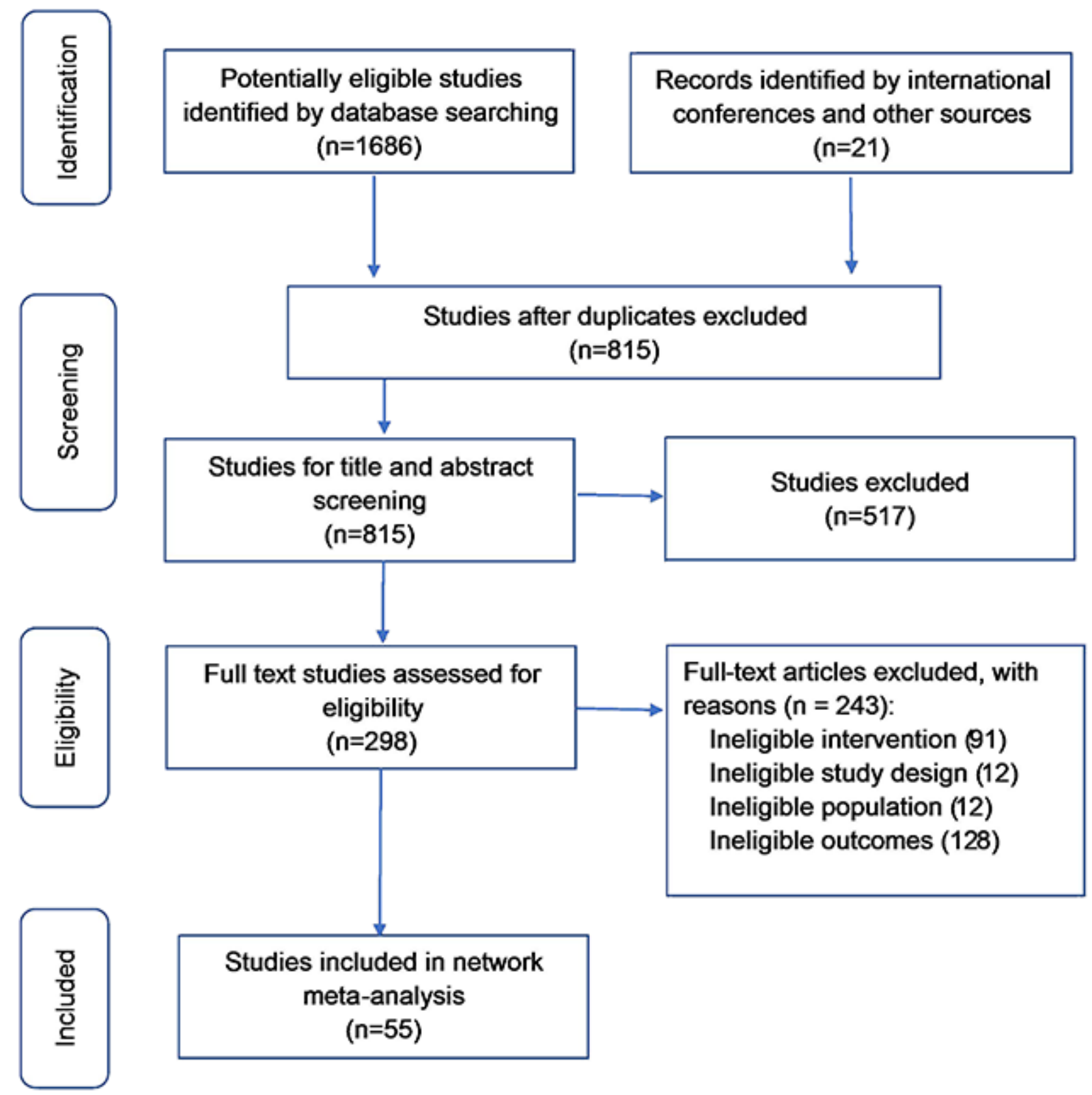

Figure 1

Study selection 

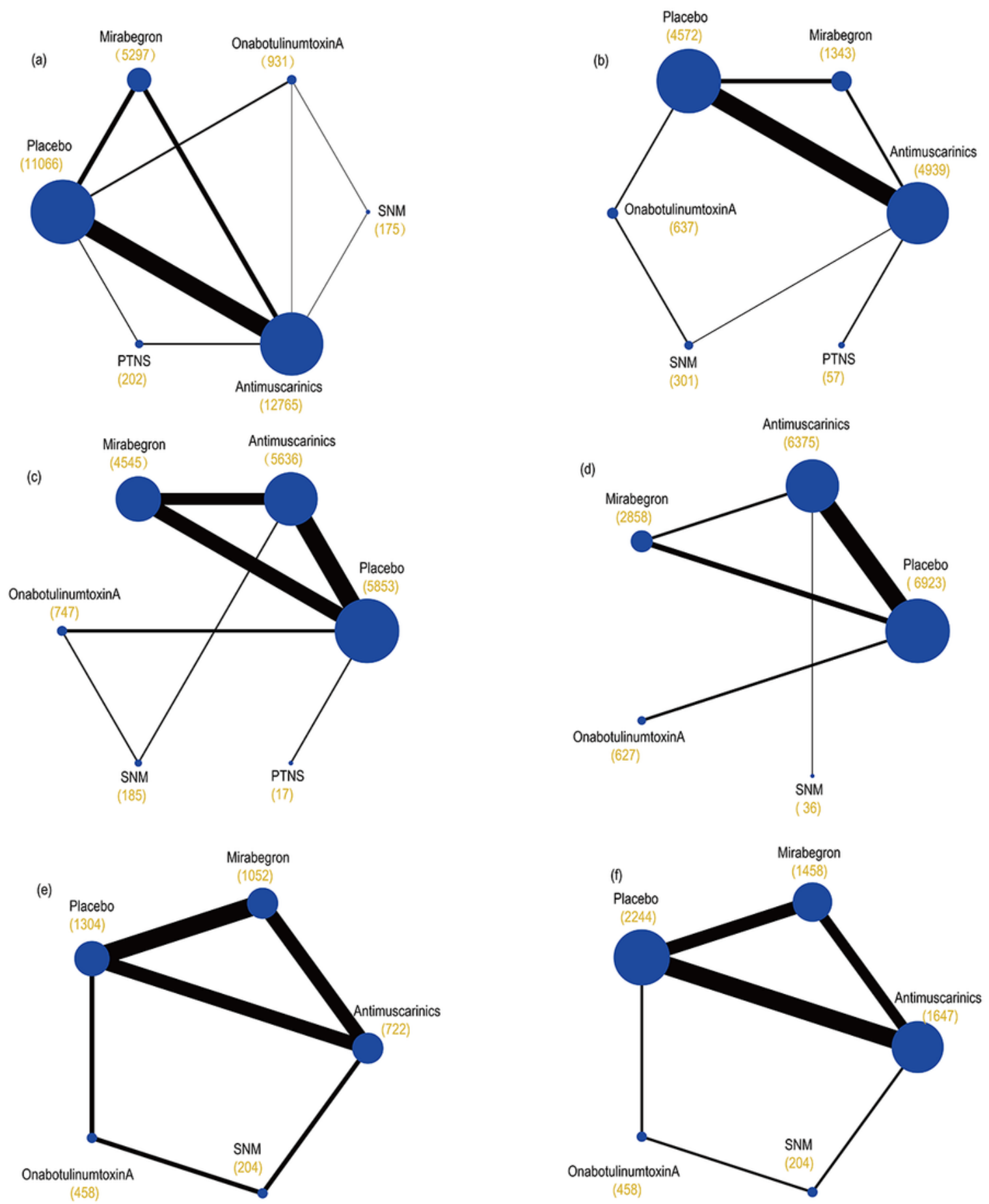

\section{Figure 2}

Network plots of: (a) mean change in the frequency of micturition/day; (b) mean change in urgency urinary incontinence episodes (UUIE)/day; (c) mean change in urinary incontinence episodes (UIE)/day; (d) mean change in urgency/day; (e) $\geq 50 \%$ reductions from baseline in urinary incontinence episodes (UIE)/day; (f) 100\% reductions from baseline in urinary incontinence episodes (UIE)/day These plots were made by Stata 16.0. Each circular node represents a type of treatment. Each line shows a type of head-to- 
head comparison. Node size and line thickness are weighted according to the number of studies evaluating each treatment and direct comparison, respectively. The total number of participants receiving a treatment is shown in brackets. SNM: sacral neuromodulation; PTNS: peripheral tibial nerve stimulation

(a)

micturition

\begin{tabular}{l|l|c|c|c|c|c|}
\hline \multirow{3}{*}{$-1.34(-2.26,-0.41)$} & $-1.04(-1.95,-0.12)$ & PTNS & $-0.10(-0.72,0.55)$ & $-0.14(-0.75,0.49)$ & $-0.89(-1.50,-0.27)$ \\
\hline$-1.49(-1.96,-1.02)$ & $-1.19(-1.63,-0.74)$ & $-0.15(-0.97,0.67)$ & Mirabegron & $-0.04(-0.21,0.12)$ & $-0.80(-0.97,-0.63)$ \\
\hline$-1.48(-1.93,-1.03)$ & $-1.18(-1.61,-0.75)$ & $-0.14(-0.95,0.67)$ & $0.01(-0.13,0.15)$ & Antimuscarinics & $-0.76(-0.87,-0.64)$ \\
\hline$-1.97(-2.42,-1.51)$ & $-1.67(-2.09,-1.24)$ & $-0.63(-1.44,0.19)$ & $-0.47(-0.61,-0.34)$ & $-0.49(-0.56,-0.41)$ & Placebo \\
\hline
\end{tabular}

(b)

UI

\begin{tabular}{|c|c|c|c|c|c|c|}
\hline \multirow{5}{*}{ urgency } & $0.28(-0.90,1.41)$ & $0.21(-1.90,2.29)$ & $-1.16(-2.60,0.23)$ & $-1.01(-2.45,0.37)$ & $-1.68(-3.10,-0.33)$ \\
\cline { 2 - 7 } & $-0.20(-1.33,0.89)$ & BoNT-A & $-0.07(-1.85,1.69)$ & $-1.43(-2.30,-0.59)$ & $-1.29(-2.15,-0.45)$ & $-1.96(-2.78,-1.17)$ \\
\cline { 2 - 6 } & & & PTNS & $-1.37(-2.97,0.23)$ & $-1.22(-2.82,0.38)$ & $-1.90(-3.47,-0.32)$ \\
\cline { 2 - 6 } & $-1.48(-2.42,-0.55)$ & $-1.28(-1.99,-0.54)$ & & Mirabegron & $0.15(-0.17,0.46)$ & $-0.53(-0.83,-0.23)$ \\
\cline { 2 - 6 } & $-1.31(-2.18,-0.43)$ & $-1.11(-1.78,-0.40)$ & & $0.17(-0.16,0.52)$ & Antimuscarinics & $-0.68(-0.95,-0.39)$ \\
\cline { 2 - 6 } & $-2.21(-3.11,-1.31)$ & $-2.00(-2.65,-1.34)$ & & $-0.73(-1.03,-0.41)$ & $-0.90(-1.11,-0.70)$ & Placebo \\
\hline
\end{tabular}

(c)

$100 \%$ reductions from baseline in UIE/day

\begin{tabular}{|c|c|c|c|c|c|}
\cline { 2 - 6 } & OnabotulinumtoxinA & $3.55(1.93,6.78)$ & $4.04(2.36,7.12)$ & $4.01(2.36,7.04)$ & $5.92(3.53,10.29)$ \\
\cline { 2 - 6 } $\begin{array}{c}\text { Z50\% reductions } \\
\text { from baseline in } \begin{array}{c}\text { in } \\
\text { UIE/day }\end{array}\end{array}$ & $1.41(0.96,2.08)$ & $\mathrm{SNM}$ & $1.14(0.57,2.24)$ & $1.13(0.57,2.19)$ & $1.67(0.84,3.25)$ \\
\cline { 2 - 6 } & $2.17(1.49,3.17)$ & $1.54(0.94,2.53)$ & Mirabegron & $0.99(0.85,1.16)$ & $1.47(1.27,1.69)$ \\
\cline { 2 - 7 } & $2.65(1.81,3.88)$ & $1.88(1.16,3.06)$ & $1.22(0.97,1.53)$ & Antimuscarinics & $1.48(1.29,1.70)$ \\
\cline { 2 - 7 } & $3.57(2.56,5.00)$ & $2.54(1.59,4.06)$ & $1.65(1.37,1.98)$ & $1.35(1.08,1.68)$ & Placebo \\
\hline
\end{tabular}

Figure 3

Network Meta-Analysis on the Outcomes of Interests Data in (a)and (b) are SMD (95\% Crl) for the comparison of row-defining treatment versus column-defining treatment. SMD less than 0 favors upperrow treatment. Data in (c) are OR $(95 \% \mathrm{Crl})$ for the comparison of row-defining treatment versus columndefining treatment. OR more than 1 favors upper-row treatment. Significant results are highlighted in bold; second line treatment are highlighted in blue; third line treatment are highlighted in yellow. SNM: sacral neuromodulation; PTNS: peripheral tibial nerve stimulation 
$\rightarrow$ Placebo $\rightarrow$ Antimuscarinics

(a)

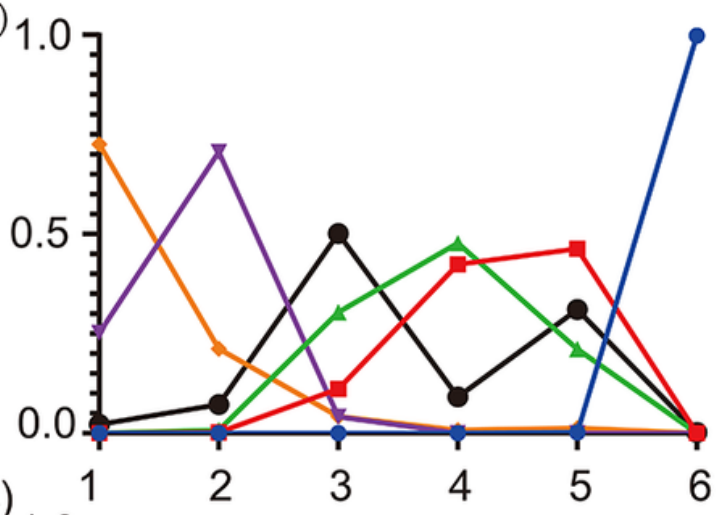

(c)

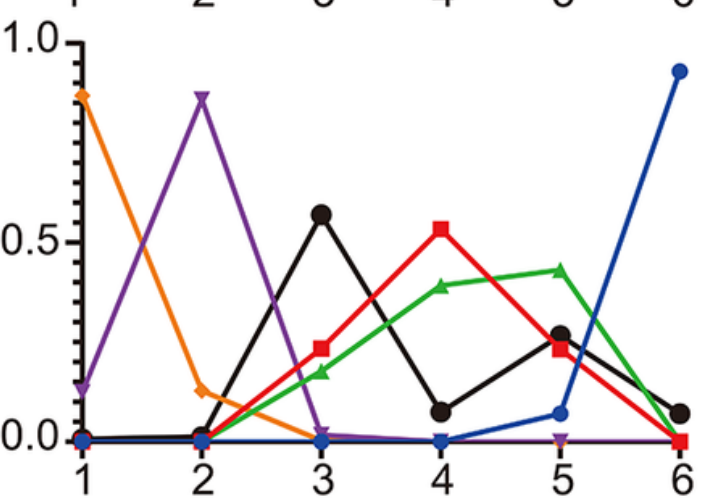

(e)

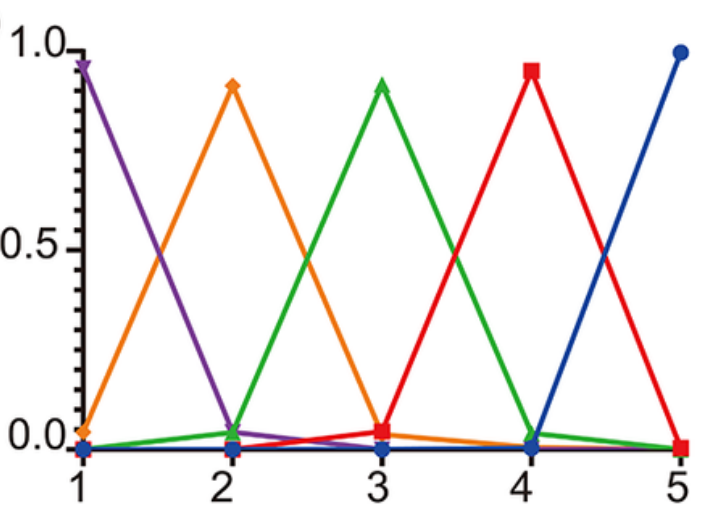

(b)

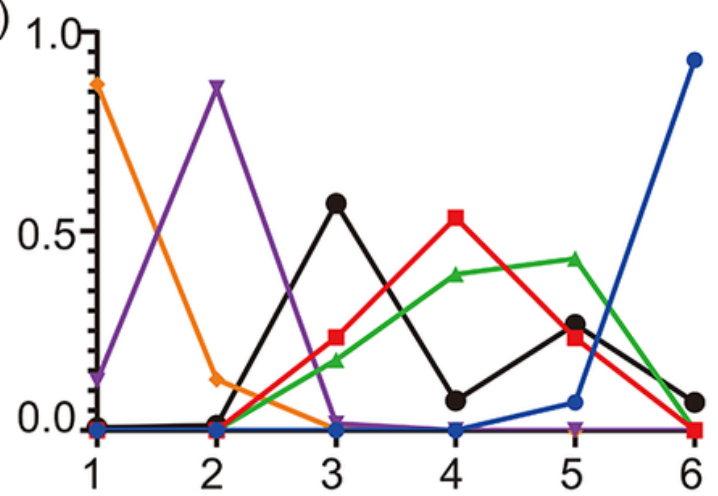

(d)

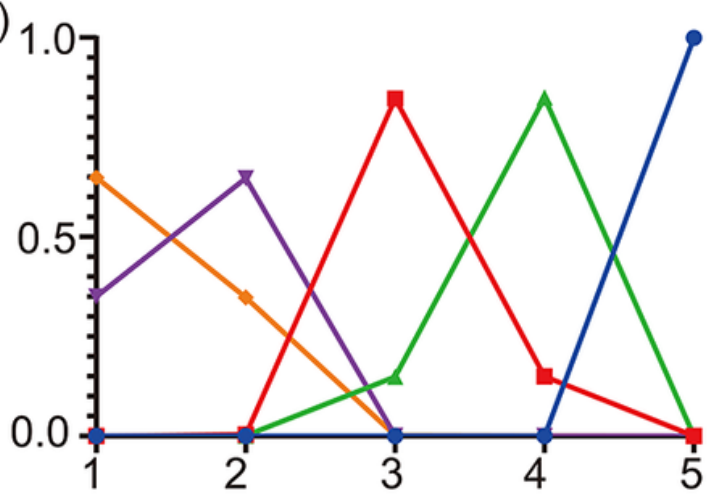

(f)

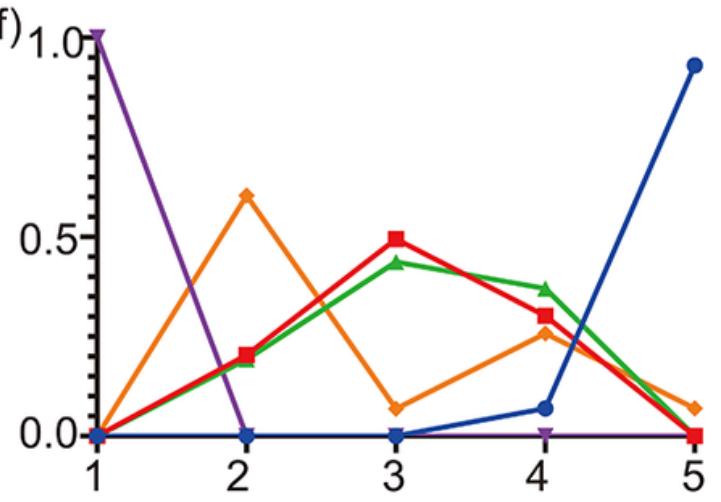

Figure 4

Rank probabilities of: (a) mean change in the frequency of micturition/day; (b) mean change in urgency urinary incontinence episodes (UUIE)/day; (c) mean change in urinary incontinence episodes (UIE)/day; (d) mean change in urgency/day; (e) $\geq 50 \%$ reductions from baseline in urinary incontinence episodes (UIE)/day; (f) 100\% reductions from baseline in urinary incontinence episodes (UIE)/day These plots were made by GraphPad Prism 8. SNM: sacral neuromodulation; PTNS: peripheral tibial nerve stimulation

\section{Supplementary Files}

This is a list of supplementary files associated with this preprint. Click to download.

- PRISMA2020checklist.docx 
- Supplementarymaterial.docx

Page 15/15 